\title{
ACTITUDES Y VALORACIÓN EN EL USO DE UNA RED SOCIAL PARA EL APRENDIZAJE DEL FRANCÉS LENGUA EXTRANJERA
}

\author{
Alfredo Álvarez Álvarez ${ }^{1}$ \\ Universidad de Alcalá. España. \\ a.alvarezalvarez@uah.es
}

\section{RESUMEN}

En este artículo se presentan los resultados más relevantes de un estudio realizado en torno a la valoración que un grupo de estudiantes del Grado de Estudios Ingleses de la Universidad de Alcalá realizaron, en la materia Francés III, del 3er curso, impartida mediante el uso de una red social. Entre otros objetivos, se pretendía valorar la percepción de los alumnos con relación a las posibilidades de uso que ofrece un entorno virtual como la red para el aprendizaje de una lengua extranjera. Para llevar a cabo la investigación, se consideró conveniente adoptar un enfoque metodológico de carácter cuantitativo, estableciendo dos líneas de trabajo diferenciadas. De un lado, se propuso una encuesta de carácter mixto, distribuida en cuatro bloques y, de otro, se planteó el curso desde la propuesta de una serie de tareas que, por su carácter de progresividad, permitieran, además de una evaluación individualizada, una valoración de carácter cuantitativo que pudiera aportar elementos de contraste con la encuesta realizada.

A grandes rasgos, los resultados confirman los datos obtenidos por otras investigaciones similares realizadas en otros contextos educativos, en lo que se refiere al aprendizaje de una lengua extranjera.

Desde esta perspectiva y, partiendo del estudio realizado, cabe señalar que se abren nuevas vías de investigación, en particular en lo que se refiere a la evaluación del proceso de aprendizaje del Francés Lengua Extranjera en un contexto TIC.

\section{PALABRAS CLAVE}

Redes sociales - blogs - itinerario - tecnologías del aprendizaje

\footnotetext{
1Alfredo Álvarez Álvarez: Doctor por la Universidad Autónoma y profesor de Lengua Francesa en la Universidad de Alcalá. Sus investigaciones se centran en el uso de las tecnologías en la enseñan de la lengua y de la traducción.
} 


\title{
ATTITUDE AND ASSESSMENT IN THE USE OF A SOCIAL NETWORK FOR LEARNING FRENCH AS A FOREIGN LANGUAGE
}

\begin{abstract}
This article presents the most relevant findings from a study on the assessment that a group of students of the Degree in English Studies of the University of Alcalá made, about the subject French III, of the third year, taught in a social network. The aim, among others, was to assess the students' perception of the possible use of a virtual environment such as the network for learning a foreign language.
\end{abstract}

For the research, it was considered appropriate to adopt a methodological, quantitative approach, establishing two different lines of work. On the one hand, a mixed survey in four blocks was proposed and, on the other hand, the course was proposed as a series of tasks which, given their progressiveness, would lead not only to individual assessment but also to a quantitative assessment that could provide elements to compare with the survey carried out.

Generally speaking, the findings confirm the data acquired from other similar research carried out in other educational contexts, in learning a foreign language.

From this perspective and based on the study carried out, it must be pointed out that new lines of research are open, in particular regarding the assessment of the learning process of French as a Foreign Language in a TIC context.

\section{KEY WORDS}

Social networks - blogs - itinerary - learning technologies

\section{INTRODUCCIÓN}

Las tecnologías en sí mismas no representan una ventaja para el aprendizaje del francés como lengua extranjera, salvo que se pueda demostrar lo contrario. Sin embargo, en los últimos años especialmente, han irrumpido en la enseñanza de diversas formas, de tal manera que hay quien apunta que, pasada la etapa de las TIC -Tecnologías de la información y la comunicación- hemos entrado en las TAC Tecnologías del aprendizaje y el conocimiento (Sotomayor, 2010). Tal vez sea pronto aún para determinar si este concepto se consolidará o no, pero sí resulta alentador que se separe de la denominación TIC, que ha dado lugar a interpretaciones erráticas, especialmente en lo que se refiere a las tecnologías en educación, una de las disciplinas más beneficiadas (Cobo y Pardo, 2007: 101), y ámbito en el cual los cambios a que han dado lugar comienzan a ser bastante profundos. Uno de ellos, sin duda de los más significativos, es el relativo al papel del profesor que, con las tecnologías, en lugar de suministrar conocimientos, participa en el proceso de generar conocimiento junto con el estudiante, de forma construida y compartida 
(Meso, K., Pérez da Silva, J.A., Mendiguren Galdospin, T, 2010). Lo que no quiere decir, lógicamente, que el estudiante quede excluido del proceso, sino que tiene en este ámbito un papel esencialmente activo convirtiéndose en el verdadero protagonista de su propio aprendizaje (Beltrán, 1996: 20).

En lo que se refiere al aprendizaje de una segunda lengua, y en particular del Francés Lengua Extranjera, las ventajas del uso de tecnologías con respecto a las metodologías tradicionales son diversas. Tomando en consideración el hecho de que los procesos centrales del aprendizaje son los procesos de organización y comprensión del material informativo, ya que el aprendizaje es el resultado de la interpretación o transformación de los materiales de conocimiento (García Sans, 2008), cabe señalar que las últimas tendencias en educación propugnan el trabajo en grupo como metodología predominante (Meso et Alii, 2010), en la cual los alumnos son los protagonistas de la actividad en el aula. La primera de las ventajas es la posibilidad de trabajar sobre documentos reales, tanto escritos como en formato audio o vídeo, a la que se une la facilidad para disponer de dichos documentos. Naturalmente, mucho más que en otras áreas de conocimiento, el uso de documentos sonoros en la enseñanza de la lengua, incluidos los audiovisuales, está en la base tanto del desarrollo de las diversas competencias lingüísticas como de la transmisión de los contenidos culturales necesarios, si pensamos que aprender una lengua no consiste únicamente en memorizar unas cuantas palabras con sus reglas de funcionamiento, sino una forma de pensar y de ver el mundo. Las tecnologías nos permiten, en definitiva, aprender en contexto.

Sin embargo, cualquier consideración acerca del aprendizaje de la lengua francesa y las tecnologías ha de pasar por concretar, en lo que se refiere a estas últimas, algunas precisiones de carácter técnico que son, de entrada, muy importantes ya que determinan en gran parte las rutas del aprendizaje. La más significativa de todas es el diferente nivel de impacto en la educación entre la web 1.0 y la web 2.0.

\subsection{El paso del Rubicón}

Según el propio O'Reilly (2005), la caída del índice Nasdaq en 2000 marcó el inicio de la tecnología web 1.0 a la 2.0 (Piscitelli, 2007). Sea como sea, lo cierto es que la web de hoy no tiene mucha relación con la que existía en 2000. Actualmente, cualquier usuario tiene a su disposición una enorme cantidad de aplicaciones que conviven entre sí dándole una mayor riqueza a su actividad de la que se podía esperar en la etapa anterior, en la que el usuario no pasaba de ser un mero espectador que consumía la información sin ninguna posibilidad de intervenir, más allá del envío-recepción del correo electrónico o de rellenar formularios ad hoc en páginas más o menos estáticas. 
De todas las herramientas que esta web 2.0 pone a disposición del profesor hay 3 que merecen una atención especial por cuanto ofrecen unas prestaciones que han propiciado que los docentes reparen en ellas como dispositivos para su uso en la clase: son los blogs, las plataformas o campus virtuales y las redes sociales.

- Los blogs. Representan uno de los dispositivos más sencillos de uso de la web 2.0. Aparecieron a finales de los 90 con Tim Berners-Lee, y a mediados de la década de los 2000 algunos docentes emprendíamos nuestras primeras experiencias con estas herramientas a las que considerábamos, con acierto, herramientas de futuro. Con ligeros matices, la clasificación que se realizó entonces de los diferentes tipos de blogs mantiene su vigencia (Álvarez: 2006): a) el blog como espacio pedagógico; b) el blog como herramienta para investigación o experiencia pedagógica; c) el blog como espacio de comunicación; y d) el blog como proyecto pedagógico de colaboración. Lo cierto es que estos dispositivos, dos décadas más tarde de su creación, siguen ofreciendo ventajas indudables, entre las que podemos destacar (Ríos, 2013):

- Facilidad de creación;

- Permiten la interacción por medio de la función Comentarios;

- Ofrecen herramientas de edición y publicación muy útiles para la práctica de una lengua extranjera.

Si bien es cierto que el concepto del espacio en el blog presenta algunos inconvenientes para el docente, entre los cuales podemos citar el hecho de que, de no establecer una organización adecuada, la profusión de contenidos propende inevitablemente al caos, no es menos cierto que el propio concepto de distribución del espacio ha sido exitosa. Prueba de ello es que ha dado pie a otros dispositivos, como las plataformas o las propias redes sociales para, a partir de esa formulación básica, construir espacios enriquecidos, más aptos para integrar aplicaciones y trabajo en grupo.

- La plataforma o Campus Virtual. Casi todas las universidades han habilitado aulas virtuales para el uso de sus propios docentes. Se trata de un entorno que permite establecer diferentes tipos de relaciones (Ríos, 2013). En general, todas las plataformas ofrecen aplicaciones parecidas en las que es frecuente encontrar un espacio dedicado a la gestión del profesor, un espacio de trabajo y un espacio de comunicación; este último con diferentes alternativas. Además, numerosas plataformas ofrecen igualmente algún espacio exclusivo para el alumno, con el fin de que pueda comunicarse en ámbitos de mayor privacidad tomando en consideración el hecho de que el profesor, si bien no puede participar, sí puede en cambio observar.

- $\quad$ Las redes sociales. Estos dispositivos no nacieron para su uso en educación aunque en muchos sentidos parecen pensadas para ello. Partiendo de la teoría de los seis grados de separación, formulada por Frigyes Karinthy, una red social puede entenderse como "un sitio en la red cuya finalidad es permitir a los usuarios relacionarse, comunicarse, compartir contenido y crear comunidades", o como una herramienta de "democratización de la información que transforma a las personas en receptores y en productores de contenidos" (Ureña, A., Ferrari, A., Blanco, D., Valdecasa, E., 2011). Se han definido también como "servicios dentro de las webs que permiten al usuario, 1) construir un perfil público o semipúblico dentro de un sistema limitado; 2) articular una lista de otros usuarios con los que comparte una conexión y 3) visualizar y rastrear su lista de contactos y las elaboradas por otros usuarios dentro del sistema. La naturaleza y nomenclatura de esas conexiones suele 
variar de una red social a otra" (Boyd, D., Ellison, N., 2007). En educación, las redes sociales han comenzado a valorarse de manera significativa ya que aportan, entre otros muchos elementos, la dimensión social del aprendizaje. Entre las ventajas que aportan podemos mencionar (Álvarez: 2009, 156):

contribuyen a compartir responsabilidades,

- aprovechan los recursos de toda la comunidad,

refuerzan la idea de comunidad como elemento común,

ayudan a establecer distintos tipos de sinergias y colaboraciones entre sus participantes.

Acaso una de las primeras variables que tengamos que considerar en el trabajo con redes sociales en educación sea la de que el profesor, en lugar de suministrar conocimientos, participa en el proceso de generar conocimiento junto con el estudiante, de forma construida y compartida (Meso et Alii, 2010). Naturalmente, desde esta óptica se entenderá que el proceso de organización junto con el de comprensión del material informativo resulten centrales (García Sans, 2008). El profesor, en palabras de Santamaría (2005) “ha dejado de ser el orador sagrado, dispensador exclusivo de la ciencia, y ya no es la única fuente de conocimientos, ya que él mismo ha de aprender de muchos otros agentes: de los medios de comunicación, de sus compañeros, de la sociedad en general y, cabe señalar igualmente, de los propios estudiantes". En todo caso, lo que sí es cierto es que la red social presenta una serie de características que la hacen atractiva para los estudiantes y útil para los profesores. Entre esas características está la posibilidad de un uso síncrono y/o asíncrono, que da lugar a diferentes niveles de comunicación; profesor-alumnos, alumnos-profesor, alumnosalumnos..., tanto en forma de mensajería como de chat, si se considera necesario. Al mismo tiempo, el hecho de poder manejar y publicar documentos de distinto formato (Word, Excel, Pdf, mp3...) incrementa las opciones de trabajo considerablemente ya que, especialmente cuando se trata del aprendizaje de una lengua, los recursos de audio y de vídeo resultan ser de una gran trascendencia para desarrollar diferentes destrezas. Este tipo de dispositivo, en definitiva, nos permite trasladar al aula situaciones de comunicación verosímiles, algo necesario en el proceso de aprendizaje de una lengua, en palabras de Boquete (2009), lo que nos conduce igualmente a la idea de aprendizaje en contexto que, entendemos, favorecen tanto las tecnologías en general como las redes sociales en particular, ya que están capacitadas para dar soporte a diferentes tipos de aplicaciones.

Por otra parte, de acuerdo con los estudios realizados por Panckhurst y Marsh (2011), "más allá de los entornos formales de aprendizaje virtual basados en instituciones (VLE) o los sistemas de gestión de aprendizaje (LMS), las redes sociales pueden resultar beneficiosas para el aprendizaje individual y en colaboración, ya que no solo proporcionan una mayor sensación de libertad en comparación con supuestas limitaciones de los VLE y LMS académicos, sino que además animan a los estudiantes a ser más independientes y a asumir una mayor responsabilidad en su propio aprendizaje, de modo que podemos entender que para los estudiantes son espacios empáticos en los que se relacionan sin dificultad y a los que se acercan con altos niveles de motivación. 


\section{OBJETIVOS}

El objetivo del presente estudio ha sido analizar la percepción de un grupo de estudiantes de lengua francesa con relación a las posibilidades de uso que ofrecen las tecnologías en su conjunto, en el aprendizaje de una lengua. Para ello se diseñó específicamente un espacio virtual en una red social de código abierto, utilizándola como centro de actuaciones a lo largo del cuatrimestre que duró el estudio.

Igualmente, mediante encuestas realizadas al principio y al final del estudio, se pretendió analizar las posibilidades de integración de una red social en el contexto de la clase y su nivel de aceptación entre los estudiantes.

\section{METODOLOGÍA}

En lo que respecta al presente estudio, los alumnos habían cursado las asignaturas cuatrimestrales Francés I y Francés II, partiendo del nivel A1 MCER. Estas asignaturas presentaban una fuerte carga lectiva presencial de ocho créditos ECTS. Todas las clases se impartieron en sala de informática, con equipos dotados de auriculares y micrófonos, a fin de tomar en consideración detalles de carácter técnico como la calidad del audio o de las grabaciones de sonido que se realizaron.

El curso se planteó sobre la base de la utilización de una red social, en este caso Ning, como recurso central en torno al cual se articularían otros:

- Libro de texto (C'est la vie! Méthode de français, niveau 4. Santillana Français). El objetivo de la utilización del libro de texto fue proporcionar al alumno una hoja de ruta que le permitiera en todo momento una orientación suficiente en la asignatura. Sin embargo, este recurso se reinterpretó en clave tecnológica, esto es, se decidió el uso de aquellas propuestas que pudieran tener una traslación a un entorno virtual.

- Página web ONYVA (www.onyva.es). Sitio web que creamos en 2004 con contenidos para el aprendizaje del francés, reconocido como base de datos por el Registro de la Propiedad Intelectual. La web dispone de un conjunto de contenidos clasificados por niveles que responde al deseo de crear, organizar y presentar un gran conjunto de recursos para el aprendizaje del Francés Lengua Extranjera.

- Canal Dailymotion. Este espacio es un repositorio de documentos de vídeo, al estilo de otros que existen en la red. Como ya se ha señalado, el recurso audiovisual debía tener una presencia importante en la medida en que representa la forma más aproximada de reproducción de los contenidos socio-lingüístico-culturales de la lengua francesa. Trabajamos con dos tipos de documentos audiovisuales: de un lado, aquellos que incorporamos a la docencia, de diversas procedencias y que se proponen como material de clase y, de otro, aquellos documentos que produjeron los propios estudiantes. Por razones de optimización de los recursos, entendimos que era más útil crear un canal para la clase que pedir a los estudiantes que cada cual crease el suyo, ya que una de las ventajas que tiene un canal colectivo es la de que el sistema ofrece al profesor diversas informaciones, como el número de reproducciones de cada uno de los documentos, cuál es el más popular... etc., es decir, proporciona parámetros significativos a la hora de 
establecer la evaluación al tiempo que sugiere pistas para futuras tareas, ya que algunos estudiantes hacen propuestas especialmente creativas que sirven al conjunto.

- Actividades de escucha. En la metodología tradicional, el audio de cada método se escuchaba en un radiocasete. En nuestro caso, preferimos utilizar la propia red para esta actividad, subiendo aquellos archivos que consideramos necesarios. De este modo se garantizó que la escucha se realizara en óptimas condiciones.

Otra de las actividades realizadas en este contexto fue la audición de emisiones de radio que ofrecen algunas emisoras francesas, convenientemente estructuradas y con opción para ejercicios de comprensión.

- Itinerarios. El itinerario es un conjunto de recursos autocorrectivos con el que culmina cada unidad y que se trabajaba básicamente en presencial, dedicándole aproximadamente una hora y media, al final de cada unidad didáctica, ya que este recurso condensa los contenidos de la unidad.

Al principio del curso todos los estudiantes pasaron un test de nivel en línea, compuesto por 50 preguntas. El interés de dicha prueba consistía en determinar el punto de partida con la mayor exactitud. Se eligió el test de Language Link porque dispone de dos elementos específicos; de un lado su carácter progresivo, especialmente apto para alumnos con el nivel que se les suponía (A2-B1) y, de otro, la presencia de un cronómetro, lo que permitía añadir un elemento muy significativo a la evaluación del nivel de lengua de cada estudiante. Además y, siempre con la intención de determinar con la mayor precisión el punto de partida, se pasó una encuesta, compuesta por 19 preguntas, con el objetivo de conocer el hábito de los estudiantes en el uso de tecnologías, su nivel de manejo y la integración que hacían de ellas en las tareas habituales del Grado (Estudios Ingleses).

En la primera clase se dieron a los estudiantes unas nociones básicas sobre el manejo de la red a utilizar y se procedió a la creación del correspondiente perfil y diseño de los espacios. Con carácter previo se había organizado la red atendiendo a una serie de parámetros que la hicieran atractiva a la vista. Estos parámetros eran el aspecto, la distribución de los espacios y la inclusión de una serie de herramientas de uso habitual. Estas, que se visualizaban en la página principal mediante enlaces, consistían en diccionarios monolingües, bilingües, de conjugación..., etc., así como diversos portales para el aprendizaje del francés. El objetivo, como ya se ha mencionado, era centralizar en la red la práctica totalidad de actividades, de forma que fuera la referencia principal para el mayor número de tareas, el recurso sobre el que girasen todos los demás. La mayoría de los estudiantes realizó todas las tareas iniciales con prontitud y, desde el primer momento, el grado de implicación fue muy alto, de lo cual se proporcionarán datos más adelante.

El curso se planteó por tareas desde una perspectiva progresiva, dando prioridad a las competencias orales, de forma que cada una de ellas pudiera ser evaluable, distinguiendo sin embargo dos clases, las tareas de carácter obligatorio y las de carácter voluntario. Cabe señalar que, desde la perspectiva mencionada, cada unidad didáctica se completaba con una presentación oral que los estudiantes conocían al inicio. En unos casos se grababa en vídeo para publicarla en la red social y en otros no. A continuación se exponen algunos ejemplos llevados a la práctica: 
- Bajo el título de Moi et ma famille se les pidió que, en un tiempo aproximado de 2 minutos, dieran toda la información posible acerca de ellos mismos y de su familia. Esta actividad tenía un doble objetivo, de un lado trabajar la cohesión en el grupo a través de la puesta en común de informaciones de carácter personal y, de otro, valorar su capacidad para elaborar y materializar un discurso.

- Bajo el título de Mon voyage idéal se les pidió que narraran, con el mayor detalle posible, cuál sería el viaje que preferirían hacer. Se pretendía con ello practicar la secuenciación de tareas: búsqueda de la información, selección, organización y presentación, ya que debían llevar a cabo la actividad sin leer documento alguno, únicamente se les permitió disponer de un pequeño guión.

- Presentación/explicación de temas gramaticales. Habida cuenta de que eran alumnos con un nivel B1, se consideró que estaban suficientemente capacitados para explicar con garantías un tema gramatical. Para ello, se hizo una distribución, al inicio del curso, de todos los temas incluidos en el programa, de tal modo que cada estudiante conocía el tema que debía explicar y la correspondiente unidad didáctica. Se hacía siempre en soporte informático (PowerPoint o blog) de forma que se pudiera publicar en la red social y quedar a disposición de los demás compañeros. Esta actividad tuvo un alto grado de implicación ya que cada estudiante puso todo su empeño en que el tema que le había correspondido fuera convenientemente comprendido por los demás en todos sus términos.

- Visionado de entre 25 y 30 vídeos, con su correspondiente explotación, para facilitar una mejor comprensión oral. Todos ellos se publicaron en la red de modo que el visionado se realizaba en clase dando un tiempo ${ }^{\circ}$ en el cual los estudiantes podían visionarlo varias veces.

- Grabación en audio (con la aplicación Vocaroo): Presentación personal con el mayor número posible de detalles. El documento grabado estaba en la red para que cualquiera pudiera escucharlo.

- Grabación en vídeo: bajo el título De ma fenêtre, se pidió cada estudiante que describiera lo que se veía desde la ventana de su habitación. Se pretendía practicar la habilidad para describir el entorno con la mayor precisión posible.

- Presentación en PPT: bajo el título Mon village se les dieron una serie de indicaciones para que, en un tiempo de entre 10-15 minutos, presentaran su pueblo/ciudad, en PowerPoint. Para ello se proporcionaron una serie de pautas que debieron cumplir (gran profusión de imágenes, muy poco texto en las diapositivas, información precisa). Las presentaciones se grabaron en vídeo y se publicaron en la red.

- Presentación oral: bajo el título de Mon partenaire idéal se pidió a los estudiantes que elaboraran un discurso con las cualidades que desearían para su ideal de pareja.

- Virelangues o trabalenguas. Esta actividad se estructuró con el objetivo de reforzar la fonética. Para ello, se asignó a cada estudiante un trabalenguas, se le envió en clase, mediante bluetooth, del móvil del profesor a cada uno de los estudiantes. La razón para que lo tuvieran en su teléfono móvil era que de esa forma "lo llevan siempre consigo" y, por tanto, podía acceder a él y memorizarlo con facilidad. Cuando el trabalenguas revestía mucha dificultad o el estudiante lo encontraba muy complicado, se hacía una grabación en audio con el propio móvil. De este modo se perfeccionaron algunos sonidos que resultan especialmente complejos para el hablante de lengua materna español (previa elección detallada de los trabalenguas).

- Lectura literaria a cámara. Para esta actividad se les pidió que eligieran una obra literaria, seleccionaran un párrafo y lo leyeran a cámara, preferiblemente buscando un primer plano lo más cercano posible. El objetivo, dado que el momento en que se realizó era aproximadamente a mitad del cuatrimestre, era evaluar la pronunciación para, una vez 
visionados los vídeos, proceder a un análisis personalizado con cada estudiante acerca de sus posibilidades de mejora.

- Lectura y explicación de informaciones concretas en diarios franceses en línea. Uno de los elementos más relevantes que aportan las tecnologías en el aula es, y se ha mencionado, el aprendizaje en contexto, que aprovecha los contenidos socioculturales para incorporarlos al proceso del aprendiz. Por ello, los diarios en su versión digital ofrecen varias ventajas, entre las que podemos citar el hecho de que se actualizan permanentemente, o el que aportan igualmente, por ser extranjeros, una perspectiva diferente sobre los mismos temas que ayuda enormemente al estudiante a situarse en el plano cultural con respecto a otras concepciones del mundo que se vehiculan a través del lenguaje.

- Audición de música francesa especialmente seleccionada de acuerdo con el nivel y la capacidad de comprensión de los estudiantes. Las nuevas generaciones tienen en la música un elemento esencial de sus vidas. De hecho, pasan una gran parte de la jornada escuchándola. Desde esta perspectiva $\mathrm{y}$, aprovechando su carácter global que nos permite acercarnos con facilidad a la que se produce en cualquier país, pensamos que, dotada del correspondiente aparato de selección y explotación, la actividad de escuchar música podía ofrecer elementos de motivación para el estudiante que lo acercaran, de un lado, a la cultura francófona y, de otro, mediante el trabajo con la letra de las canciones, atender a aspectos específicos de desarrollo de diversas competencias.

- Presentaciones orales: Qué seré en el futuro, Si je n'étais pas espagnol, Le bonheur. Estas presentaciones formaron parte del conjunto de actividades orales dirigidas al desarrollo de diversas destrezas, entre las cuales la expresión oral era un objetivo prioritario.

- Inserciones en la red social (posts): en este caso, se trataba de dotar a la red de un aspecto más específico de comunicación. Por ello, se eligió la fórmula de los posts, que los estudiantes utilizaron para hacer aportaciones al conjunto, como informaciones de carácter diverso (becas, convocatorias), propuestas, opiniones, intercambios, etc.

- Redacción y grabación en vídeo de un informativo imaginario. El objetivo de la actividad era darle una continuidad lógica a la serie de actividades que se habían realizado en los medios de comunicación, especialmente en la prensa escrita. Se trataba de redactar, organizar y grabar en vídeo un informativo tomado del modelo de la TV.

- Creación y desarrollo de una unidad didáctica para el aprendizaje de la lengua francesa. Este era el trabajo final y, para poder realizarlo, se impartieron 2 sesiones de aprendizaje del programa eXe Learning, con el que desarrollaron la tarea.

- Aportaciones voluntarias. En los primeros días del curso se explicó a los estudiantes que, además de las tareas obligatorias $\mathrm{y}$, por tanto, calificables, cabría igualmente la posibilidad de realizar tareas de carácter voluntario, igualmente calificables. Para ello se propuso una pauta que consistió en plantear, con carácter periódico, una presentación individual de dichas aportaciones, en la cual cada estudiante pudiera explicar a los demás el porqué de cada una de las aportaciones que había hecho y, de ahí, se compartieran. En su mayoría se trató de vídeos musicales en los que los estudiantes reflejaban sus gustos y preferencias. Las presentaciones gozaron de un enorme atractivo ya que todos mostraron mucho interés por conocer las motivaciones de los compañeros en incluir tal o cual contenido. 


\section{RESULTADOS}

En este apartado haremos referencia a dos orientaciones diferentes. De un lado, los resultados obtenidos en cuanto al trabajo propio de los estudiantes $y$, de otro, el que se deduce de la encuesta realizada.

En cuanto a las tareas ejecutadas por los estudiantes, constituyeron un total de 276, con 19 para cada uno. Además de ello, se realizó el test de nivel mencionado anteriormente más 4 ejercicios de control (bilans) que se proponían en el método de clase. El resultado alcanzado en lo que se refiere al nivel de realización de tareas puede considerarse positivo, ya que se situó en el 94,6\%, dentro de una gran homogeneidad, pues el número de tareas propuestas y no realizadas es tan solo de 15, es decir, corresponde a una proporción de 1 tarea no realizada por estudiante, lo que representa un 5,4\%. En otro orden de cosas, el número de posts voluntarios representó un 19\% del total y correspondió a una media de 5,4 posts por estudiante. En el gráfico 1 pueden verse estos parámetros en su conjunto.

\section{Gráfico 1: Resultados}

\begin{tabular}{ll}
$\begin{array}{l}\text { Tareas obligatorias } \\
\text { (individualmente) }\end{array}$ & 19 \\
$\begin{array}{l}\text { Tareas efectivamente } \\
\text { realizadas }\end{array}$ & $\begin{array}{l}261 \\
(94.6 \%)\end{array}$ \\
Tareas totales no realizadas & $\begin{array}{l}15 \\
(5,4 \%)\end{array}$ \\
$\mathrm{N}^{\circ}$ total de posts & 341 \\
$\mathrm{~N}^{0}$ de posts obligatorios & 276 \\
$\mathrm{~N}^{0}$ de posts voluntarios & 65 \\
\hline Media de posts voluntarios & 5,4 \\
\hline
\end{tabular}

Por lo que hace referencia a la encuesta realizada, esta se estructuró en 4 bloques: a) Manejo del ordenador, b) Mejora en el aprendizaje de la lengua francesa, c) Apreciación de la red social, y d) Valoración del aprendizaje de la lengua con el uso de TIC. En el primer bloque se trataba de conocer la percepción de los estudiantes con relación al manejo del ordenador y saber con qué tipo de usuario se identificaban. El resultado arrojó una gran mayoría (11/15) que consideraron que sus conocimientos estaban dentro del rango usuario. Además, se produjo una respuesta en la dirección bastante alto y otra en especializado. Esta primera percepción proporciona una valiosa información, ya que muestra, más allá de la realidad objetiva, cómo cada estudiante se percibe a sí mismo cuando se sitúa frente a la máquina 
ordenador. Por otra parte, se tiende a pensar que los estudiantes, por el hecho de ser nativos digitales, deben considerarse usuarios avanzados o muy avanzados, lo cual no siempre es así, ya que tienden a restringir el uso del ordenador a determinadas funciones básicas, como búsqueda de información (a veces con métodos muy rudimentarios) y gestión de sus perfiles en las redes sociales, incluyendo la publicación de comentarios y/o fotos.

En el segundo bloque (Mejora en el aprendizaje de la lengua), la media de respuestas para la variable "En qué aspectos consideras que has mejorado a lo largo de este cuatrimestre" estuvo bastante igualada para las opciones "manejo del ordenador", "conocimiento de fuentes informáticas", "aprendizaje de procesos de trabajo", "conocimiento de recursos pedagógicos", "familiarización con entornos on line" / la puntuación más alta la obtuvo el ítem "conocimiento de recursos pedagógicos" con 3,92 puntos de media, y el más bajo de los ítems formulados en positivo "manejo del ordenador" con 3 puntos de media. El ítem "no he mejorado" era un ítem formulado en negativo y se puntuó muy bajo (0,43 de media).

En lo relativo a la pregunta "cita los aspectos en los que has mejorado, si no están en la lista anterior", los alumnos marcaron siguientes respuestas (ya que se trataba de una pregunta abierta): (las opiniones están expuestas tal y como están en los cuestionarios, sin clasificar): Manejo de programas que no había manejado con anterioridad. Perfeccionamiento en el manejo de otros que ya conocía y manejaba/Conocimiento de muchos programas y Webs interesantes para llevar a cabo los trabajos/Conocimiento de nuevos programas/El aprendizaje de, entre otros, crear actividades pedagógicas autocorregibles lo considero muy útil para nuestra futura vida laboral - en el caso de la enseñanza - pues autonomiza y dinamiza el trabajo de estudiante.

En lo que respecta a la adquisición y desarrollo de destrezas, las puntuaciones que los estudiantes asignaron eran superiores, quedando los niveles más elevados en Expresión oral (valoración: 4.25/5), Comprensión oral (valoración: 4.16/5) y Comprensión audiovisual (valoración: 4.08/5). Cabe señalar que la opción No he mejorado, incluida en las respuestas, obtuvo una calificación de 0 puntos. Todos los respondientes valoraron la experiencia como positiva y ninguno consideró que no había mejorado en las competencias lingüísticas indicadas.

En el tercer bloque (Apreciación de la red social) se trataba de que los estudiantes valorasen la red social utilizada para el curso. La variable "Cómo valoras la utilización de una red social (NING) como herramienta para la clase de Francés Lengua Extranjera" presentaba como opciones de respuesta la posibilidad de marcar o no la opción que se considerara válida desde el punto de vista del alumno. Para este tipo de experiencia, la opción más nombrada en este caso fue que la utilización de la red NING es motivadora (8/15); las opciones "no aporta nada al aprendizaje" y "complicada" no tuvieron puntuación. La opción "otras" presentaba algunas descripciones, fueron las siguientes: Te ayuda a estar al día con la asignatura, y trabajas de una manera más amena/El espíritu de compartir nuestros progresos, conocimientos e inquietudes sobre la materia es altamente favorable pues tienen un aspecto motivador para el alumno. Poder enseñar tu trabajo y ver el de los demás fomenta una actitud colaborativa útil en la enseñanza sobre todo de una lengua extranjera. En cuanto a las "Ventajas que has 
visto en la utilización de la red social Ning, esta presentaba una valoración muy positiva (la totalidad de alumnos la marcaron) para la opción "estimula el trabajo cooperativo", también recibió una valoración positiva (7/12) la opción de que NING "abre las posibilidades de trabajo en equipo", algunos alumnos marcaron la opción "ayuda a mejorar las relaciones personales" y sólo uno "establece vínculos profesionales". Las opciones "no aporta gran cosa" y "entorpece la clase" no fueron marcadas por ninguno de los alumnos. La pregunta abierta que se refiere a las Observaciones que se pueden aportar en esta variable son siguientes: (sin clasificar) Puedes observar el trabajo de tus compañeros, y subir cosas que te parecen útiles y puedes ayudar al resto/Me parece práctica útil y entretenida. No da una apariencia complicada ni aburrida como Blackboard/Establece vínculos entre estudiantes, útil para estar al día.

En lo relativo al cuarto bloque (Valoración del aprendizaje de la lengua con el uso de TIC), a partir de los datos de estudiantes para la variable "Tu sensación, después de esta experiencia de trabajo con las TIC, es de que..." podemos ver que la mayoría de los alumnos están de acuerdo que "son más entretenidas" (10 sujetos), en el segundo lugar queda la opción "con ellas se aprende mejor" (7 sujetos), y en el tercer lugar queda la opción "con ellas se aprende más rápido" (5 sujetos), las opciones “entorpecen el aprendizaje" y "son iguales que otros métodos" no fueron marcadas.

En el caso de la pregunta "Otros (puedes aportar lo que creas conveniente" los estudiantes prefirieron no opinar.

Para la variable "Consideras esta experiencia de aprendizaje en la clase de Francés" podemos observar siguientes datos: las opciones más marcadas entre las ofrecidas han sido: "original" - 10 sujetos; "muy útil" - 8 sujetos; "la recomendaría" 6 sujetos; "útil" - 4 sujetos. Las opciones "como todas" y "no me ha gustado" no fueron marcadas por ningún estudiante; Entre las respuestas a la pregunta abierta Otras opciones (puedes aportar lo que creas conveniente) se escribió lo siguiente: $\mathrm{He}$ aprendido más que ningún año.

Entre los aspectos de enseñanza aprendizaje con TICs que parecieron más útiles opinaron todos los alumnos encuestados, señalando lo siguiente (sin clasificar): El poder enviar las cosas y trabajar desde casa y con el ordenador facilita mucho las cosas/Las presentaciones en clase, la utilización de la plataforma por poder compartir con todos el trabajo y así comparar/Me ha parecido útil la utilización de Francés en ligne, creo que es una propuesta interesante para poner muchos conocimientos en común, te permite ver tus errores y tus progresos/Los recursos de Internet han sido muy útiles porque hay infinidad de vídeos y documentos de audio para practicar/Las grabaciones de audio, más que las visuales porque así te concentras más en el audio, la plataforma y sobre todo el aprender a hacer páginas web/Aprender a manejar nuevos programas de ordenador/Aprender a utilizar nuevos programas de ordenador $y$ de Internet/Las grabaciones para mejorar nuestra expresión $y$ pronunciación corrigiéndonos a nosotros mismos y los itinerarios que cumplen las competencias requeridas necesarias/Trabajar con ordenadores para utilizar el material audiovisual (vídeos, tanto grabados por nosotros como publicados en Internet), me parece que ameniza el proceso de aprendizaje/Fácil estar al día y comparar los trabajos con los compañeros, muy útil para la relación entre estudiantes y el trabajo en equipo/El uso, sobre 
todo, de los itinerarios, esto nos permite trabajar dentro y fuera del aula todos los aspectos de Francés como lengua extranjera. El hecho de tenerlos online nos permite desde casa acceder a ejercicios autocorregibles donde podemos ser nosotros quienes vayamos comprobando nuestra audición en la lengua. La estructuración por unidades y apartados es muy útil, pues ayuda a enfocar nuestra actividad a aquellos aspectos que queramos trabajar.

\section{DISCUSIÓN}

Si bien se considera que el uso de las TIC aporta elementos de novedad en lo que se refiere a aplicación de metodologías, se realizó un estudio acerca de la percepción del aprendizaje del FLE mediante el uso de una red social, algo que, entre otras ventajas, presenta la de que el aprendizaje se realiza en contexto. Para ello, una vez analizadas diversas posibilidades para dotar al estudio de elementos de contraste, se pusieron en práctica una serie de dispositivos, ordenándose todos en torno a la red social. Las razones de esta elección tienen que ver con el hecho de que, de acuerdo con las características de los alumnos, se consideró que una red ofrecía un entorno suficientemente empático, debido a razones de proximidad, facilidad de manejo, e inmediatez, que podían representar aspectos muy motivadores para el aprendizaje y, al mismo tiempo, estimular el avance en destrezas específicas, de acuerdo con el MCER. Además, se diseñaron específicamente una serie de tareas encaminadas a desarrollar las destrezas orales. Los resultados obtenidos permiten afirmar que los estudiantes se sintieron muy identificados con la red social y con la metodología propuesta, considerándose una herramienta muy adecuada para el aprendizaje de lenguas extranjeras y, en concreto, el francés. Por otra parte, la red social, por el hecho de estar creada en francés y representar un entorno "culturalmente francés" puede considerarse un espacio de aprendizaje que recoge los elementos socioculturales de la lengua. Además, la versatilidad que ofrece, así como la visibilidad del entorno estimulan la participación y el learning by doing, ya que cada miembro de la red se aprovecha de las contribuciones de los demás, constituyéndose por tanto un verdadero entorno de aprendizaje.

Entre los argumentos en los que se sustenta esta apreciación están las propias valoraciones realizadas por los estudiantes, que consideraron la red motivadora y muy útil, además de que estimula el trabajo cooperativo. Por otra parte, las tecnologías son consideradas por ellos como originales y entretenidas. De hecho, entre los comentarios hay bastantes referencias a la pertinencia del trabajo cooperativo y al hecho de que la observación del trabajo de los compañeros ha contribuido al aprendizaje.

En todo caso, el estudio ha de continuarse en el futuro tratando de orientar la investigación, a la vista de los datos obtenidos, en la línea de la calidad y rapidez del aprendizaje del FLE mediante el uso de herramientas tecnológicas. Faltan por determinar instrumentos de medición del aprendizaje de forma que se pueda caminar, partiendo de la hipótesis de que las TIC mejoran la percepción del aprendizaje añadiendo una motivación suplementaria, en la línea de medir hasta donde sea posible ese aprendizaje. 


\section{REFERENCIAS}

Álvarez Álvarez, A. (2006) Le blog, un outil du futur pour le présent, Cahiers de l'Apliut, Vol. XXV No $3 \perp 2006$ : Nouvelles formes d'écriture et de lecture dans l'enseignement des langues de spécialité.

Álvarez Âlvarez, A. (2009) Nuevas tecnologías para la clase de francés: teoría y práctica. Quiasmo Editorial. Madrid.

Beltrán Llera, J. A. (1996): Procesos, estrategias y técnicas de aprendizaje. Madrid: Editorial Síntesis, S.A.

Boquete, G. La integración de destrezas (2009): Recuperado de http:// www.slideshare.net/Gabinoboquete/integracin-de-destrezas-2691559.

Consultado el 06/05/2014.

Boyd, D. y Ellison, N. (2007). Social Network Sites: Definition, history and scholarship, Journal of Computer-Mediated Communication.

Cobo Romaní, C., Pardo Kuklinski, H. (2007). Planeta Web 2.0. Inteligencia colectiva o medios fast food. Grup de Recerca d'Interaccions Digitals, Universitat de Vic. Flasco México. Barcelona-México DF. E-book de acceso gratuito. Versión 0.1. Web oficial del libro: http://www.planetaweb2.net/

García Sans, A. (2008). Las redes sociales como herramientas para el aprendizaje colaborativo: una experiencia con Facebook. Recuperado de http:// www.mentalidadweb.com/wpcontent/uploads/2008/07/comunicacion_f acebook_annagarciasans.pdf. Consultado el 09/05/2014.

Langage Link, (ND) Test. Recuperado de http://www.french.language.ru/french/onlinetest/index.html. Consultado el 09/05/2014.

Meso Ayerdi, K./ Pérez Dasilva, J.A./ Mendiguren Galdospin, T. (2010) Las redes sociales como herramientas para el aprendizaje colaborativo. Presentación de un caso desde la UPV/EHU. Alfabetización mediática y culturas digitales. Universidad de Sevilla.

O'Reilly, T. (2005) What Is Web 2.0. Design Patterns and Business Models for the Next Generation of Software. Recuperado de http://www.oreilly.com/. Consultado el $07 / 05 / 2014$.

Piscitelli, A. (2007) La inflación lingüística llama Web 2.0, Planeta Web 2.0. Inteligencia colectiva o medios fast food [en línea]. Grup de Recerca d'Interaccions Digitals, Universitat de Vic. Flasco México. Barcelona-México DF. E-book de acceso gratuito. Versión 0.1-Septiembre de 2007. [20-01-2008] Web oficial del libro: http:/ / www.planetaweb2.net/

Ríos Guardiola, G. (2013) Recursos virtuales para el aprendizaje del francés como lengua extranjera. Perspectivas y limitaciones pedagógicas. Educación XX1, vol. 16, núm. 1.

Santamaría González, F. (2005): Herramientas colaborativas para la enseñanza usando tecnologías web: weblogs, wikis, redes sociales y web 2.0. Recuperado de http:// desarrollo.uces.edu.ar:8180/dspace/bitstream/123456789/597/1/Herrami entas_colaborativas_web.2.pdf . Consultado el 03/05/2014.

Sotomayor, G. (2010). Las redes sociales como entornos de aprendizaje colaborativo mediado para segundas lenguas (L2). EDUTEC, Revista Electrónica de Tecnología 
Educativa. Núm. 34. Diciembre 2010. Recuperado de http:/ / edutec.rediris.es/revelec2/revelec34/. Consultado el 14/05/2014.

Ureña, A., Ferrari, A., Blanco, D., Valdecasa, E. (2011) Las redes sociales en Internet. ONTSI (Observatorio Nacional de las Telecomunicaciones y de la SI. Fondo Europeo de Desarrollo Regional.

\section{AUTOR}

\section{Alfredo Álvarez Álvarez}

Alfredo Álvarez Álvarez es profesor de Lengua Francesa en la Universidad de Alcalá (Madrid). Doctor por la Universidad Autónoma de Madrid, con una tesis sobre recursos en internet para el aprendizaje y la traducción del francés, sus trabajos de investigación versan sobre la integración de las tecnologías en clase, tanto en lo que respecta a la enseñanza de la lengua francesa como de su traducción. Sobre el tema ha realizado numerosas publicaciones, de las que puede mencionarse el libro Nuevas tecnologías para la clase de francés: teoría y práctica. 